\title{
Metanálise bayesiana de parâmetros genéticos para características de crescimento em bovinos de corte
}

\author{
Juliana Di Giorgio Giannotti(1), Irineu Umberto Packer(2), Maria Eugênia Zerlotti Mercadante(3) \\ e Roseli Aparecida Leandro(4)
}

\begin{abstract}
(1)Instituto de Economia Agrícola, Secretaria da Agricultura e Abastecimento, Av. Miguel Stéfano, 3900, CEP 04301-903 São Paulo, SP. E-mail: juliana@iea.sp.gov.br (2)Escola Superior de Agricultura Luiz de Queiroz (Esalq), Dep. de Zootecnia, Caixa Postal 9, CEP 13418-900 Piracicaba, SP. E-mail: iupacker@esalq.usp.br (3)Instituto de Zootecnia, Estação Experimental de Zootecnia de Sertãozinho, Caixa Postal 63, CEP 14160-970 Sertãozinho, SP. E-mail: mercadante@iz.sp.gov.br ${ }^{(4)}$ Esalq, Dep. de Ciências Exatas. E-mail: raleandr@esalq.usp.br
\end{abstract}

\begin{abstract}
Resumo - Foi conduzida uma metanálise, sob enfoque bayesiano, em um conjunto de estimativas de herdabilidade, para características de crescimento em bovinos de corte de raças indianas, com 869 estimativas de herdabilidade de efeito direto, 186 estimativas de herdabilidade de efeito materno e 123 estimativas do coeficiente de correlação genética entre os efeitos direto e materno. A construção e o desenvolvimento de um modelo hierárquico possibilitam a obtenção de estimativas combinadas de herdabilidade para as características peso ao nascimento, peso ao desmame, peso aos 365 dias e peso aos 550 dias, cujos valores são, respectivamente: 0,31, 0,24, 0,28 e 0,33 para efeitos diretos; 0,09, 0,13, 0,12 e 0,05 para efeitos maternos; e -0,16, -0,16, -0,20 e -0,16 para as correlações entre esses efeitos. A metanálise conduzida sob enfoque bayesiano mostrou-se bastante adequada, uma vez que um modelo hierárquico considera as variâncias dentro e entre os estudos, e sua implementação e condução são facilitadas em virtude, principalmente, do avanço conseguido na área computacional.
\end{abstract}

Termos para indexação: inferência bayesiana, estimativas combinadas, herdabilidade, correlação genética.

\section{Bayesian meta-analysis of genetic parameters for growth traits in beef cattle}

\begin{abstract}
Bayesian meta-analysis was performed in a data set of heritability estimates for growth traits of zebu beef cattle with 869 direct heritabilities, 186 maternal heritabilities, and 123 direct-maternal genetic correlation. The fitting and development of a hierarchical model made possible to obtain pooled heritability estimates for birth weight, weaning weight, weight at 365 days of age and weight at 550 days of age, whose values were, respectively: $0.31,0.24,0.28$ e 0.33 for direct effects; $0.09,0.13$, 0.12 e 0.05 for maternal effects; and $-0.16,-0.16$, -0.20 e -0.16 for direct-maternal genetic correlation. Meta-analysis conducted under Bayesian framework was adequate, since hierarchical model considers between-study and within-study variances, and its implementation and conduction are facilitated, mainly, due to the advance in computational area.
\end{abstract}

Index terms: Bayesian inference, pooled estimates, heritability, genetic correlation.

\section{Introdução}

O uso de artigos científicos referentes a um mesmo assunto, com a mesma metodologia de análise e o mesmo conjunto de dados, porém atualizado, é uma prática que pode ocorrer em pesquisa aplicada, até mesmo no melhoramento genético animal. Nesse contexto, é grande o número de artigos publicados sobre estimativas de herdabilidade de efeito direto e materno, e a correlação entre esses efeitos, nas características de crescimento. Conscientes do grande número de estimativas de herdabilidade de efeitos direto e materno, e suas correlações, presente em artigos publicados, Koots et al. (1994a, 1994b), Mercadante et al. (1995) e Lôbo et al.
(2000) realizaram, independentemente, revisões sobre esses parâmetros genéticos, para características de crescimento, em bovinos de corte de diferentes raças. $\mathrm{O}$ foco de análise desses autores foi conhecer o comportamento biológico dessas características de produção e verificar qual sua contribuição genética e ambiental. Para tanto, os autores utilizaram valores médios conjuntos de diferentes trabalhos publicados com essas estimativas.

A amplitude de variação dos resultados, presente na literatura, e o grande número de artigos publicados sobre um mesmo assunto, são os principais argumentos para pesquisadores obterem valores médios. Como exemplo, tem-se a abrangência dos resultados obtidos 
de estimativas de herdabilidade de efeito direto. Para a característica peso ao nascimento, Tawah et al. (1993) encontraram o valor de 0,65; ao avaliar o desmame, Sakaguti (2000) e Plasse et al. (2002) encontraram, respectivamente, os valores de 0,08 e 0,47 ; Ribeiro et al. (2001) e Ferraz-Filho (2002) encontraram, respectivamente, os valores de 0,40 e 0,17 para peso aos 365 dias, e em relação ao peso aos 550 dias, os valores de 0,76 e 0,13. Características com estimativas de herdabilidade abaixo de 0,20 são consideradas pouco herdáveis, já aquelas com valores acima de 0,40 , são consideradas altamente herdáveis (Bourdon, 2000). Assim, prevalece a dúvida, em todas as características, quanto a serem pouco ou muito herdáveis.

Ainda mais discutíveis são as estimativas de correlação entre os efeitos direto e materno, publicadas para as características de crescimento, principalmente até um ano de idade, como pode ser visto nas características: peso ao nascimento e ao desmame, em que Tawah et al. (1993) encontraram os valores de -0,93 e -0,68, respectivamente; peso aos 365 dias, em que Eler et al. (1994) e Ferraz-Filho (2002) encontraram, respectivamente, os valores de -0,91 e -0,20; peso aos 550 dias, em que Ribeiro et al. (2001) e Ferraz-Filho (2002) encontraram, respectivamente, os valores de $-1,00$ e $-0,28$. Assim, a fim de nortear uma conclusão, uma média obtida de resultados já existentes pode ser uma solução interessante.

Existe o desafio de se desenvolver metodologias efetivas para comparar, e possivelmente combinar, informações de trabalhos relacionados, a fim de se realizar alguma inferência confiável sobre determinado tema. Assim, é crescente o número de estudos sobre a implementação e a melhoria de procedimentos estatísticos para a aplicação da metanálise, cujo trabalho pioneiro, no Brasil, foi efetuado por Giannotti et al. (2002).

O principal foco de crítica para a aplicação da metanálise é quanto à variabilidade existente entre os estudos a serem combinados. Assim, modelos de metanálise, que incorporam na análise o componente da variância entre os estudos, são amplamente discutidos tanto na teoria clássica quanto na bayesiana. Porém, autores como Larose \& Dey (1997) e Gelman et al. (2004) consideram que a formulação de um modelo bayesiano hierárquico oferece mecanismo natural, para descrever e explicar a heterogeneidade existente entre os estudos individuais, enquanto a teoria clássica para condução da metanálise envolve a combinação da magnitude de efeitos. O modelo bayesiano hierárquico permite que relações probabilísticas a respeito dos parâmetros e hiperparâmetros, que são os parâmetros das distribuições dos parâmetros, sejam incorporadas ao modelo por meio de distribuições a priori hierárquicas.

Outras duas vantagens apresentadas pelos métodos bayesianos, sobre os clássicos na aplicação da metanálise, são que os primeiros oferecem uma estrutura conveniente para a incorporação de informações disponíveis a priori, e são aplicáveis a problemas da combinação de estudos com tamanhos amostrais pequenos, o que não ocorre quando se utilizam métodos assintóticos dos estimadores de máxima verossimilhança (Larose \& Dey, 1997). Assim, as inferências a serem conduzidas, observando-se os resultados da análise, serão, possivelmente, mais precisas do que aquelas obtidas pelo método clássico.

Este trabalho teve como objetivo combinar estimativas publicadas de parâmetros genéticos, por meio da metanálise, utilizando modelo bayesiano hierárquico para as características de crescimento, peso ao nascimento (PN), peso ao desmame (PD), peso aos 365 dias (P365) e peso aos 550 dias (P550), em bovinos de corte, de origem indiana.

\section{Material e Métodos}

Os dados utilizados referem-se a 869 estimativas de herdabilidade de efeito direto $\left(\hat{\mathrm{h}}_{\mathrm{d}}^{2}\right), 186$ estimativas de herdabilidade de efeito materno $\left(\hat{\mathrm{h}}_{\mathrm{m}}^{2}\right)$ e 123 estimativas do coeficiente de correlação genética entre os efeitos direto e materno $\left(\mathrm{r}_{\mathrm{dm}}\right)$, das características de crescimento peso ao nascimento (PN), peso ao desmame (PD), peso aos 365 dias (P365) e peso aos 550 dias (P550), obtidas em populações de bovinos de corte, de origem indiana, que são: 182 de $\hat{\mathrm{h}}_{\mathrm{d}}^{2}$, 33 de $\hat{\mathrm{h}}_{\mathrm{m}}^{2}$ e 27 de $\mathrm{r}_{\mathrm{dm}}$ para PN; 331 de $\hat{\mathrm{h}}_{\mathrm{d}}^{2}$, 81 de $\hat{\mathrm{h}}_{\mathrm{m}}^{2}$ e 57 de $\mathrm{r}_{\mathrm{dm}}$ para PD; 153 de $\hat{\mathrm{h}}_{\mathrm{d}}^{2}$, 34 de $\hat{\mathrm{h}}_{\mathrm{m}}^{2}$ e 22 de $\mathrm{r}_{\mathrm{dm}}$ para P365 e 203 de $\hat{\mathrm{h}}_{\mathrm{d}}^{2}$, 38 de $\hat{\mathrm{h}}_{\mathrm{m}}^{2}$ e 17 de $\mathrm{r}_{\mathrm{dm}}$ para P550. Essas estimativas procedem de 186 artigos publicados entre os anos de 1968 e 2003, dos quais 102 estão presentes no trabalho de revisão realizado por Mercadante et al. (1995), e 84 provêm de pesquisa bibliográfica realizada para a atualização desses dados, do ano de 1995 até 2003. Tal pesquisa englobou anais de congressos, teses, dissertações e bases de dados como CAB e AGRIS, em que as palavras-chave utilizadas foram: "nelore"; "nellore"; "zebu”; "Bos indicus”; e "heritabili*”.

A informação da variância associada a cada valor de estimativa de herdabilidade é essencial em uma metanálise. As variâncias das estimativas de herdabilidade foram calculadas por meio da metodologia descrita por Koots et al. (1994a) e Falconer \& Mackay (1996). Os valores de $\hat{\mathrm{r}}_{\mathrm{dm}}$ foram transformados para a 
quantidade z, conforme descrito por Giannotti et al. (2002), que é recomendado pelo fato de o valor $\mathrm{z}_{\mathrm{i}}$ apresentar distribuição normal, com variância igual a $\operatorname{var}\left(\mathrm{z}_{\mathrm{i}}\right)=1 /\left(\mathrm{n}_{\mathrm{i}}-3\right)$, em que $\mathrm{n}_{\mathrm{i}}$ é o número de observações do i-ésimo estudo. Assim, para combinar os $\hat{r}_{\mathrm{dm}}$, foram utilizadas as variâncias de $\mathrm{z}$.

Uma forma natural de descrever o problema e analisar os dados em questão é utilizar um modelo bayesiano hierárquico. $\mathrm{Na}$ análise bayesiana hierárquica, os parâmetros das distribuições dos parâmetros, chamados hiperparâmetros, são desconhecidos, mas com uma distribuição conhecida (Gelman et al., 2004). Assim, o modelo hierárquico para a realização da metanálise teve a seguinte estrutura, assumindo-se $s_{i}^{2}$ conhecido:

$\mathrm{y}_{\mathrm{i}} \mid \alpha_{\mathrm{i}}, \mathrm{s}_{\mathrm{i}}^{2} \sim \operatorname{Normal}\left(\alpha_{\mathrm{i}}, \mathrm{s}_{\mathrm{i}}^{2}\right)$

$\alpha_{\mathrm{i}} \mid \mu, \tau^{2} \sim \operatorname{ind} \operatorname{Normal}\left(\mu, \tau^{2}\right)$;

em que $\mathrm{y}_{\mathrm{i}}$ é a estimativa a ser combinada, correspondente ao i-ésimo estudo $(\mathrm{i}=1, \ldots, \mathrm{I})$ (representada por $\hat{\mathrm{h}}_{\mathrm{di}}^{2}$ para a i-ésima herdabilidade de efeito direto, ou $\hat{\mathrm{h}}_{\mathrm{mi}}^{2}$ para a i-ésima herdabilidade de efeito materno, ou $\mathrm{z}_{\mathrm{i}}$ para o i-ésimo coeficiente de correlação, transformado entre as herdabilidades de efeito direto e materno, nas quatro características); $\alpha_{\mathrm{i}}$ é o valor verdadeiro da estimativa a ser combinada $\left(\mathrm{h}_{\mathrm{di}}^{2}\right.$, ou $\mathrm{h}_{\mathrm{mi}}^{2}$, ou $\mathrm{z}_{\mathrm{i}}$ ), correspondente ao i-ésimo estudo; $\mathrm{s}_{\mathrm{i}}^{2}$ é a variância da estimativa a ser combinada, ou seja, a variância presente dentro do i-ésimo estudo (representada por $\operatorname{var}\left(\hat{\mathrm{h}}_{\mathrm{di}}^{2}\right)$ para a variância de $\hat{\mathrm{h}}_{\mathrm{di}}^{2}$, ou $\operatorname{var}\left(\hat{\mathrm{h}}_{\mathrm{mi}}^{2}\right)$ para a variância de $\hat{\mathrm{h}}_{\mathrm{mi}}^{2}$, ou $\operatorname{var}\left(\mathrm{z}_{\mathrm{i}}\right)$ para a variância de $\mathrm{z}_{\mathrm{i}}$, nas quatro características); $\mu$ é a estimativa combinada (representada por $\hat{\mathrm{h}}_{\mathrm{d}+}^{2}$ para herdabilidade de efeito direto, ou $\hat{\mathrm{h}}_{\mathrm{m}+}^{2}$ para herdabilidade de efeito materno, ou $\hat{r}_{\mathrm{dm}+}$ para coeficiente de correlação entre as herdabilidades de efeito direto e materno, nas quatro características); $\tau^{2}$ é a variância existente entre os estudos, $\tau^{2}>0$ (representada por $\hat{\tau}_{\mathrm{d}}^{2}$ para os estudos com herdabilidade de efeito direto, $\hat{\tau}_{\mathrm{m}}^{2}$ para os estudos com herdabilidade de efeito materno, ou $\hat{\tau}_{\mathrm{dm}}^{2}$ para estudos com os coeficientes direto e materno, nas quatro características).

A partir do modelo especificado anteriormente, a função de verossimilhança para $\alpha_{1}, \alpha_{2}, \ldots, \alpha_{\mathrm{I}}$, é dada por:

$$
\begin{aligned}
& \mathrm{L}\left(\alpha_{1}, \ldots, \alpha_{\mathrm{I}}\right)=\prod_{\mathrm{i}=1}^{\mathrm{I}} \frac{1}{\sqrt{2 \pi \mathrm{s}_{\mathrm{i}}}} \exp \left\{\frac{-1}{2 \mathrm{~s}_{\mathrm{i}}^{2}}\left(\mathrm{y}_{\mathrm{i}}-\alpha_{\mathrm{i}}\right)^{2}\right\} \\
& \times \prod_{\mathrm{i}=1}^{\mathrm{I}} \frac{1}{\sqrt{2 \pi \tau}} \exp \left\{\frac{-1}{2 \tau^{2}}\left(\alpha_{\mathrm{i}}-\mu\right)^{2}\right\} \\
& \propto \exp \left\{\frac{-1}{2 \mathrm{~s}_{\mathrm{i}}^{2}} \sum_{\mathrm{i}=1}^{\mathrm{I}}\left(\mathrm{y}_{\mathrm{i}}-\alpha_{\mathrm{i}}\right)^{2}\right\} \times \tau^{-\mathrm{I}} \exp \left\{\frac{-1}{2 \tau^{2}} \sum_{\mathrm{i}=1}^{\mathrm{I}}\left(\alpha_{\mathrm{i}}-\mu\right)^{2}\right\} .
\end{aligned}
$$

No modelo sugerido anteriormente, as distribuições a priori são especificadas da seguinte maneira:

$\mu \sim \operatorname{Normal}(a, b$,$) ;$

$\tau^{2} \sim$ Gama Inversa(c, d),

em que, nos dados em questão, os valores foram a $=0$; $b=10^{-6} ; c=0,001$ e $d=0,001$. Tais valores foram empregados, pois se optou pela utilização de prioris não informativas e a razão principal para utilizá-las baseiase no fato de as inferências não serem afetadas por informações externas aos dados (Gelman et al., 2004).

Assim, as distribuições a priori para os hiperparâmetros $\mu$ e $\tau^{2}$ são, respectivamente, dadas por:

$$
\begin{aligned}
& \mathrm{p}(\mu)=\frac{1}{\sqrt{2 \pi \mathrm{b}^{1 / 2}}} \exp \left\{\frac{-1}{2 \mathrm{~b}}(\mu-\mathrm{a})^{2}\right\} \propto \exp \left\{\frac{-1}{2 \mathrm{~b}}(\mu-\mathrm{a})^{2}\right\} ; \\
& \mathrm{p}\left(\tau^{2}\right)=\frac{\mathrm{d}^{\mathrm{c}}}{\Gamma(\mathrm{c})}\left(\tau^{2}\right)^{-(\mathrm{c}+1)} \exp \left\{\frac{-\mathrm{d}}{\tau^{2}}\right\} \propto\left(\tau^{2}\right)^{-(\mathrm{c}+1)} \exp \left\{\frac{-\mathrm{d}}{\tau^{2}}\right\} .
\end{aligned}
$$

Portanto, assumindo-se independência, a distribuição a priori conjunta para $\alpha_{1}, \alpha_{2}, \ldots, \alpha_{\mathrm{I}}, \mu$ e $\tau^{2}$, é dada por:

$$
\begin{aligned}
& \mathrm{p}\left(\alpha_{1}, \ldots, \alpha_{\mathrm{I}}, \mu, \tau^{2}\right) \propto \tau^{-\mathrm{I}} \exp \left\{\frac{-1}{2 \tau^{2}} \sum_{\mathrm{i}=1}^{\mathrm{I}}\left(\alpha_{\mathrm{i}}-\mu\right)^{2}\right\} \\
& \times \exp \left\{\frac{-1}{2 b}(\mu-\mathrm{a})^{2}\right\} \times\left(\tau^{2}\right)^{-(\mathrm{c}+1)} \exp \left\{\frac{-\mathrm{d}}{\tau^{2}}\right\} .
\end{aligned}
$$

Combinando-se a distribuição a priori conjunta com a função de verossimilhança, a distribuição a posteriori conjunta é dada por:

$$
\begin{aligned}
& \mathrm{p}\left(\alpha_{1}, \ldots, \alpha_{\mathrm{I}}, \mu, \tau^{2} \mid \text { Dados }\right) \propto \exp \left\{\frac{-1}{2 \mathrm{~s}_{\mathrm{i}}^{2}} \sum_{\mathrm{i}=1}^{\mathrm{I}}\left(\mathrm{y}_{\mathrm{i}}-\alpha_{\mathrm{i}}\right)^{2}\right\} \tau^{-\mathrm{I}} \\
& \times \exp \left\{\frac{-1}{2 \tau^{2}} \sum_{\mathrm{i}=1}^{\mathrm{I}}\left(\alpha_{\mathrm{i}}-\mu\right)^{2}\right\} \times \exp \left\{\frac{-1}{2 \mathrm{~b}}(\mu-\mathrm{a})^{2}\right\} \\
& \times\left(\tau^{2}\right)^{-(\mathrm{c}+1)} \exp \left\{\frac{-\mathrm{d}}{\tau^{2}}\right\} .
\end{aligned}
$$

A partir da distribuição a posteriori conjunta, as distribuições marginais a posteriori para os parâmetros $\alpha_{\mathrm{i}}$ (i = $1,2, \ldots, I), \mu$ e $\tau^{2}$ são: 
$\mathrm{p}\left(\tau^{2} \mid\right.$ Dados $)=\int \ldots \int \mathrm{p}\left(\alpha_{1}, \ldots, \alpha_{\mathrm{I}}, \mu, \tau^{2} \mid \operatorname{Dados}\right) \mathrm{d} \alpha_{1} \ldots \mathrm{d} \alpha_{\mathrm{I}} \mathrm{d} \mu ;$ $\mathrm{p}(\mu \mid$ Dados $)=\int \ldots \int \mathrm{p}\left(\alpha_{1}, \ldots, \alpha_{\mathrm{I}}, \mu, \tau^{2} \mid\right.$ Dados $) \mathrm{d} \alpha_{1} \ldots \mathrm{d} \alpha_{\mathrm{I}} \mathrm{d} \tau^{2} ;$ $\mathrm{p}\left(\alpha_{\mathrm{i}} \mid\right.$ Dados $)=\int \ldots \int \mathrm{p}\left(\alpha_{(-\mathrm{i})}, \mu, \tau^{2} \mid\right.$ Dados $) \mathrm{d} \alpha_{-\mathrm{i}} \mathrm{d} \mu \mathrm{d} \tau^{2} ;$ $\alpha_{-i}=\left(\alpha_{1}, \ldots, \alpha_{i-1}, \alpha_{i+1}, \ldots, \alpha_{1}\right)$.

A parte computacional deste trabalho, ou seja, a obtenção das médias, variâncias e intervalos de credibilidade, foi desenvolvida utilizando-se o programa WinBUGS (Spiegelhalter et al., 2004), cujas principais características são: acomodar uma ampla classe de modelos; possibilitar que os modelos sejam especificados de maneira concisa; e construir e amostrar a distribuição a posteriori conjunta (Gilks et al., 1994). Na condução da análise, foi gerada uma cadeia com 55 mil valores para cada estimativa a ser combinada ( $\hat{\mathrm{h}}_{\mathrm{d}}^{2}$ ou $\hat{\mathrm{h}}_{\mathrm{m}}^{2}$ ou $\mathrm{z}$ ), dentro de cada uma das quatro características em estudo, PN, PD, P365 e P550. Os primeiros 5 mil valores foram descartados ("burn-in” de 5 mil), e os demais 50 mil valores foram selecionados de $10 \mathrm{em}$ 10 ("thin" de 10), assegurando-se, deste modo, a independência da amostra.

A indicação de convergência para os hiperparâmetros de interesse $\mu$ (representado por $\hat{\mathrm{h}}_{\mathrm{d}}^{2}, \hat{\mathrm{h}}_{\mathrm{m}}^{2}$ e z) e $\tau^{2}$ (representado por $\hat{\tau}_{\mathrm{d}}^{2}, \hat{\tau}_{\mathrm{m}}^{2}$ e $\hat{\tau}_{\mathrm{dm}}^{2}$ ) foi verificada tanto graficamente, por meio da análise de comportamento das trajetórias das cadeias, quanto a partir dos diagnósticos de convergência Geweke e Raftery \& Lewis, disponíveis no pacote computacional R (R Development Core Team, 2003), na "library" CODA (Best et al., 2004).

\section{Resultados e Discussão}

O algoritmo de amostragem utilizado na condução da inferência bayesiana foi o de Gibbs, de acordo com informação fornecida pelo programa WinBUGS (Spiegelhalter et al., 2004).

Nas Figuras 1 e 2, observam-se as formas das distribuições a posteriori para os hiperparâmetros $\tau^{2} \mathrm{e}$


Figura 1. Distribuições a posteriori do hiperparâmetro $\tau^{2}$ representado por: (a) $\hat{\tau}_{\mathrm{d}}^{2}$ para PN; (b) $\hat{\tau}_{\mathrm{m}}^{2}$ para PN; (c) $\hat{\tau}_{\mathrm{dm}}^{2}$ para PN; (d) $\hat{\tau}_{\mathrm{d}}^{2}$ para PD; (e) $\hat{\tau}_{\mathrm{m}}^{2}$ para PD; (f) $\hat{\tau}_{\mathrm{dm}}^{2}$ para PD; (g) $\hat{\tau}_{\mathrm{d}}^{2}$ para P365; (h) $\hat{\tau}_{\mathrm{m}}^{2}$ para P365; (i) $\hat{\tau}_{\mathrm{dm}}^{2}$ para P365; (j) $\hat{\tau}_{\mathrm{d}}^{2}$ para P550; (k) $\hat{\tau}_{\mathrm{m}}^{2}$ para P550; (l) $\hat{\tau}_{\mathrm{dm}}^{2}$ para P550. 
$\mu$, nas quatro características em estudo. As distribuições a posteriori, estimadas nos métodos bayesianos, podem ser interpretadas considerando-se que elas representam uma aproximação da verdadeira distribuição de probabilidade dos parâmetros, tendo sido observados os dados. A convergência da distribuição dos hiperparâmetros foi diagnosticada gráfica e numericamente. $\mathrm{O}$ diagnóstico gráfico foi feito por meio das amostras geradas pelo algoritmo de Gibbs (Figuras 1 e 2); na Figura 1 a distribuição a priori de $\tau^{2}$ foi a Gamainversa (c, d) e na Figura 2 a distribuição a priori de $\mu$ foi Normal (a,b), conforme visto anteriormente. Numericamente a convergência foi diagnosticada conforme proposto por Geweke e Raftery \& Lewis (Best et al., 2004).

Na Tabela 1, estão expostas a variância entre os estudos com herdabilidade de efeito direto $\left(\hat{\tau}_{\mathrm{d}}^{2}\right)$, a variância entre os estudos com herdabilidade de efeito materno $\left(\hat{\tau}_{m}^{2}\right)$ e a variância entre os estudos com coefi- ciente de correlação entre efeitos direto e materno $\left(\hat{\tau}_{\mathrm{dm}}^{2}\right)$, com respectivos intervalos de credibilidade, para as características PN, PD, P365 e P550.

Na Tabela 2, estão expostas as estimativas combinadas de herdabilidade para efeito direto $\left(\hat{\mathrm{h}}_{\mathrm{d}+}^{2}\right)$ e materno $\left(\hat{\mathrm{h}}_{\mathrm{m}+}^{2}\right)$, e coeficientes de correlação combinados entre os efeitos diretos e maternos $\left(\hat{\mathrm{r}}_{\mathrm{dm}+}\right)$, com respectivos intervalos de credibilidade, para as características PN, PD, P365 e P550.

Foram revisados trabalhos publicados sobre estimativas de herdabilidade de características de crescimento que apresentaram os componentes genéticos direto e materno. O componente genético direto dessas características é relativo aos efeitos dos genes para crescimento do próprio indivíduo sobre o seu desempenho, enquanto o componente materno é relativo aos efeitos dos genes da mãe para habilidade materna, principalmente para a produção de leite, que influenci-
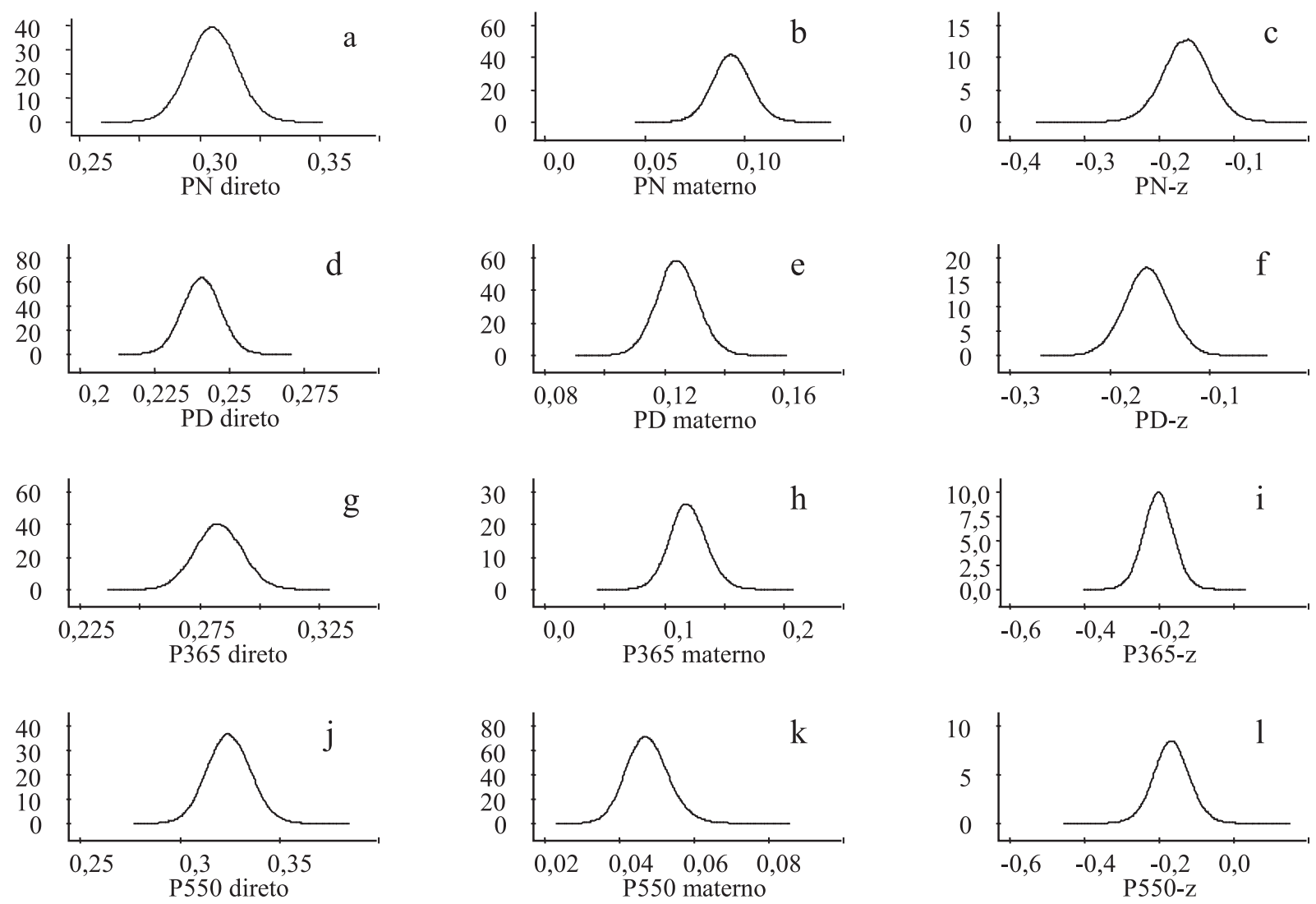

Figura 2. Distribuições a posteriori do hiperparâmetro $\mu$ representado por: (a) $\hat{\mathrm{h}}_{\mathrm{d}}^{2}$ para PN; (b) $\hat{\mathrm{h}}_{\mathrm{m}}^{2}$ para PN; (c) z para PN; (d) $\hat{h}_{d}^{2}$ para PD; (e) $\hat{h}_{m}^{2}$ para PD; (f) z para PD; (g) $\hat{h}_{d}^{2}$ para P365; (h) $\hat{h}_{m}^{2}$ para P365; (i) z para P365; (j) $\hat{h}_{d}^{2}$ para P550; (k) $\hat{\mathrm{h}}_{\mathrm{m}}^{2}$ para P550; (l) z para P550. 
am o desempenho dos bezerros por meio do ambiente oferecido por ela. Das quatro características de crescimento consideradas, aquelas mais influenciadas pela habilidade materna são o PD e o P365, que concordam com o que foi obtido neste trabalho, ou seja, valores mais elevados para as $\hat{\mathrm{h}}_{\mathrm{m}+}^{2}$ nessas características, e mais baixos nas características PN e P550, que são menos influenciadas pela habilidade materna.

Os valores (Tabela 2) de $\hat{\mathrm{h}}_{\mathrm{m}+}^{2}$ indicam que $9 \%$ para PN, 13\% para PD, 12\% para P365 e 5\% para P550, da variância entre os desempenhos dos indivíduos, ocorrem em conseqüência de genes de efeito aditivo, que determinam a habilidade materna de suas mães. Como pôde ser observado, os valores das $\hat{h}_{\mathrm{m}+}^{2}$ são mais baixos que os das $\hat{\mathrm{h}}_{\mathrm{d}+}^{2}$, o que indica que as características de crescimento estudadas são mais influenciadas por efeitos genéticos que atuam no crescimento do próprio bezerro, do que pelos efeitos genéticos para habilidade materna de sua mãe.

Tabela 1. Resumo a posteriori para o parâmetro $\tau^{2}$, representado por $\hat{\tau}_{\mathrm{d}}^{2}$ (variância entre os estudos de herdabilidade de efeito direto), $\hat{\tau}_{\mathrm{m}}^{2}$ (variância entre os estudos de herdabilidade de efeito materno) e $\hat{\tau}_{\mathrm{dm}}^{2}$ (variância entre os estudos de coeficiente de correlação entre efeitos direto e materno), nas características peso ao nascimento (PN), peso ao desmame (PD), peso aos 365 dias (P365) e peso aos 550 dias (P550).

\begin{tabular}{cccc}
\hline Característica & Parâmetro & $\begin{array}{c}\text { Média a } \\
\text { posteriori }\end{array}$ & $\begin{array}{c}\text { Intervalo de } \\
\text { credibilidade 95\% }\end{array}$ \\
\hline PN & $\hat{\tau}_{\mathrm{d}}^{2}$ & 0,013 & $(0,009 ; 0,017)$ \\
& $\hat{\tau}_{\mathrm{m}}^{2}$ & 0,003 & $(0,001 ; 0,005)$ \\
& $\hat{\tau}_{\mathrm{dm}}^{2}$ & 0,027 & $(0,015 ; 0,047)$ \\
\hline PD & $\hat{\tau}_{\mathrm{d}}^{2}$ & 0,009 & $(0,008 ; 0,012)$ \\
& $\hat{\tau}_{\mathrm{m}}^{2}$ & 0,003 & $(0,002 ; 0,005)$ \\
& $\hat{\tau}_{\mathrm{dm}}^{2}$ & 0,029 & $(0,019 ; 0,042)$ \\
\hline P365 & $\hat{\tau}_{\mathrm{d}}^{2}$ & 0,010 & $(0,008 ; 0,014)$ \\
& $\hat{\tau}_{\mathrm{m}}^{2}$ & 0,007 & $(0,004 ; 0,013)$ \\
& $\hat{\tau}_{\mathrm{dm}}^{2}$ & 0,038 & $(0,020 ; 0,070)$ \\
\hline P550 & $\hat{\tau}_{\mathrm{d}}^{2}$ & 0,017 & $(0,013 ; 0,022)$ \\
& $\hat{\tau}_{\mathrm{m}}^{2}$ & 0,0006 & $(0,0002 ; 0,001)$ \\
& $\hat{\tau}_{\mathrm{dm}}^{2}$ & 0,041 & $(0,020 ; 0,084)$ \\
\hline
\end{tabular}

Os valores obtidos para as $\hat{r}_{\mathrm{dm}+}$ foram semelhantes nas quatro características, ou seja, negativos e baixos, o que sugere associação fraca entre os componentes direto e materno. Entretanto, numerosos estudos, em particular sobre características de crescimento pré-desmame de bovinos de corte e ovinos, têm encontrado correlação antagônica entre os efeitos genéticos direto e materno, freqüentemente de $-0,5$ ou mais altas, e tais estimativas têm sido vistas com justificado ceticismo (Meyer, 1997). Para se obter boas estimativas, são necessários dados de várias gerações de seleção, com boas ligações entre elas; e para obter boas estimativas da habilidade materna das filhas de um touro, esse animal deve ter filhas com progênies. Há, freqüentemente, insuficientes ligações entre as gerações, e isto tem sido a causa de muitas estimativas publicadas, de coeficientes de correlação entre os efeitos diretos e maternos, serem viesadas (Schaeffer, 1999). Em muitos casos, as $\hat{\mathrm{h}}_{\mathrm{d}}^{2}$ e $\hat{\mathrm{h}}_{\mathrm{m}}^{2}$ são subestimadas, enquanto os efeitos de ambiente permanente materno são superestimados.

Tabela 2. Resumo a posteriori para o parâmetro $\mu$, representado por $\hat{\mathrm{h}}_{\mathrm{d}+}^{2}$ (estimativa combinada para efeito direto), $\hat{\mathrm{h}}_{\mathrm{m}+}^{2}$ (estimativa combinada para efeito materno) e $\hat{\mathrm{r}}_{\mathrm{dm}+}$ (estimativa combinada para correlação entre os efeitos direto e materno), nas características peso ao nascimento (PN), peso ao desmame (PD), peso aos 365 dias (P365) e peso aos 550 dias (P550).

\begin{tabular}{cccc}
\hline Característica & Parâmetro & $\begin{array}{c}\text { Média a } \\
\text { posteriori }\end{array}$ & $\begin{array}{c}\text { Intervalo de } \\
\text { credibilidade } 95 \%\end{array}$ \\
\hline PN & $\hat{\mathrm{h}}_{\mathrm{d}+}^{2}$ & 0,31 & $(0,29 ; 0,33)$ \\
& $\hat{\mathrm{h}}_{\mathrm{m}+}^{2}$ & 0,09 & $(0,08 ; 0,11)$ \\
& $\hat{\mathrm{r}}_{\mathrm{dm}+}$ & $-0,16$ & $(-0,22 ;-0,10)$ \\
\hline PD & $\hat{\mathrm{h}}_{\mathrm{d}+}^{2}$ & 0,24 & $(0,23 ; 0,25)$ \\
& $\hat{\mathrm{h}}_{\mathrm{m}+}^{2}$ & 0,13 & $(0,11 ; 0,14)$ \\
& $\hat{\mathrm{r}}_{\mathrm{dm}+}$ & $-0,16$ & $(-0,20 ;-0,12)$ \\
\hline P365 & $\hat{\mathrm{h}}_{\mathrm{d}+}^{2}$ & 0,28 & $(0,26 ; 0,30)$ \\
& $\hat{\mathrm{h}}_{\mathrm{m}+}^{2}$ & 0,12 & $(0,09 ; 0,15)$ \\
& $\hat{\mathrm{r}}_{\mathrm{dm}+}$ & $-0,20$ & $(-0,28 ;-0,12)$ \\
\hline P550 & $\hat{\mathrm{h}}_{\mathrm{d}+}^{2}$ & 0,33 & $(0,30 ; 0,35)$ \\
& $\hat{\mathrm{h}}_{\mathrm{m}+}^{2}$ & 0,05 & $(0,04 ; 0,06)$ \\
& $\hat{\mathrm{r}}_{\mathrm{dm}+}$ & $-0,16$ & $(-0,26 ;-0,07)$ \\
\hline & & &
\end{tabular}


Mohiuddin (1993) ao utilizar como ponderação o inverso do desvio-padrão, de dentro dos estudos, para combinar as $\hat{\mathrm{h}}^{2}$ para características de crescimento em bovinos de corte de raças européias e cruzamentos, obteve: $\hat{\mathrm{h}}_{\mathrm{d}+}^{2}$ de $0,30, \hat{\mathrm{h}}_{\mathrm{m}+}^{2}$ de 0,10 e $\hat{\mathrm{r}}_{\mathrm{dm}+}$ de -0,35 para PN; $\hat{\mathrm{h}}_{\mathrm{d}+}^{2}$ de $0,22, \hat{\mathrm{h}}_{\mathrm{m}+}^{2}$ de 0,13 e $\hat{\mathrm{r}}_{\mathrm{dm}+}$ de -0,15 para PD; $\hat{\mathrm{h}}_{\mathrm{d}+}^{2}$ de 0,31, $\hat{\mathrm{h}}_{\mathrm{m}+}^{2}$ de 0,11 e $\hat{\mathrm{r}}_{\mathrm{dm}+}$ de -0,26 para P365. Koots et al. (1994a) ao utilizar como ponderação o inverso da variância, de dentro dos estudos, para combinar as $\hat{\mathrm{h}}^{2}$ para características de crescimento em bovinos de corte de raças zebuínas, européias e cruzamentos, obtiveram: $\hat{\mathrm{h}}_{\mathrm{d}+}^{2}$ de $0,31 \pm 0,003$ e $\hat{\mathrm{h}}_{\mathrm{m}+}^{2}$ de $0,14 \pm 0,002$ para $\mathrm{PN}$; $\hat{\mathrm{h}}_{\mathrm{d}+}^{2}$ de $0,24 \pm 0,002$ e $\hat{\mathrm{h}}_{\mathrm{m}+}^{2}$ de $0,13 \pm 0,002$ para PD; $\hat{\mathrm{h}}_{\mathrm{d}+}^{2}$ de 0,33 $\pm 0,004$ para P365. Mercadante et al. (1995) ao utilizar como ponderação o inverso do desvio-padrão, dentro dos estudos, para combinar $\hat{h}^{2}$ para características de crescimento em bovinos de corte de raças zebuínas, obtiveram: $\hat{\mathrm{h}}_{\mathrm{d}+}^{2}$ de $0,33, \hat{\mathrm{h}}_{\mathrm{m}+}^{2}$ de 0,12 e $\hat{\mathrm{r}}_{\mathrm{dm}+}$ de $-0,37$ para PN; $\hat{\mathrm{h}}_{\mathrm{d}+}^{2}$ de $0,22, \hat{\mathrm{h}}_{\mathrm{m}+}^{2}$ de 0,18 e $\hat{\mathrm{r}}_{\mathrm{dm}+}$ de -0,23 para PD; $\hat{\mathrm{h}}_{\mathrm{d}+}^{2}$ de $0,20, \hat{\mathrm{h}}_{\mathrm{m}+}^{2}$ de 0,16 e $\hat{\mathrm{r}}_{\mathrm{dm}+}$ de $-0,30$ para P365; $\hat{\mathrm{h}}_{\mathrm{d}+}^{2}$ de $0,38, \hat{\mathrm{h}}_{\mathrm{m}+}^{2}$ de 0,05 para P550. Comparando-se os valores encontrados neste trabalho, com aqueles presentes nos artigos de Mohiuddin (1993), Koots et al. (1994a) e Mercadante et al. (1995), verifica-se que os valores obtidos nessas revisões foram próximos aos encontrados neste trabalho, mesmo tendo sido utilizadas metodologias e raças distintas (exceto em Mercadante et al., 1995).

Com relação aos aspectos computacionais da condução da metanálise sob o enfoque bayesiano, o programa WinBUGS (Spiegelhalter et al., 2004) mostrou-se satisfatório, em virtude de os resultados obtidos serem consistentes, e de a programação utilizada ser relativamente simples e estar bastante documentada no próprio módulo de ajuda do programa e em artigos e livros (Gilks et al., 1994; Smith \& Spiegelhalter, 1996; Normand, 1999; Gelman et al., 2004).

Recomenda-se, assim, a obtenção de valores combinados de estimativas de herdabilidade de efeito direto, materno e suas correlações, para as características de crescimento em bovinos de corte de origem indiana, por meio da metanálise bayesiana, uma vez que por meio dessa metodologia é possível comparar e reunir resultados de estudos distintos, para se alcançar uma conclusão geral sobre o corpo da pesquisa.
Valores combinados, obtidos neste trabalho, podem revelar mais sobre as estimativas de herdabilidade e correlação genética de rebanhos zebuínos, do que uma única estimativa obtida de apenas um trabalho publicado. Tais valores podem também fornecer subsídios para a realização de inferências, indicar novas hipóteses a serem testadas em estudos futuros e servir de valores iniciais em programas de melhoramento animal.

\section{Conclusões}

1. A utilização do modelo bayesiano hierárquico é bastante conveniente em metanálise, pois leva em consideração as variâncias dentro e entre os estudos.

2. As estimativas combinadas de herdabilidade e correlação de características de crescimento são coerentes e apresentam intervalos de credibilidade adequados.

\section{Agradecimentos}

Ao CNPq, pelo financiamento deste estudo.

\section{Referências}

BEST, N.G.; COWLES, M.K.; VINES, S.K. CODA manual version 0.30. Disponível em: <http://www.mrc-bsu.cam.ac.uk/bugs/ documentation/coda03/node9.html>. Acesso em: 2 jun. 2004.

BOURDON, G.E.P. Understanding animal breeding. Upper Saddle River: Prentice-Hall, 2000. 538p.

ELER, J.P.; FERRAZ, J.B.S.; LÔBO, R.B.; JOSAKIAN, L.A. Genetic antagonism between growth and maternal ability in nelore cattle. Revista Brasileira de Genética, v.17, p.59-64, 1994.

FALCONER, D.S.; MACKAY, T.F.C. Introduction to quantitative genetics. Edinburgh: Addison Wesley Longman, 1996. 464p.

FERRAZ-FILHO, P.B.; RAMOS, A. de A.; SILVA, L.O.C. da; SOUZA, J.C. de; ALLENCAR, M.M. de; MALHADO, C.H. Tendência genética dos efeitos direto e materno sobre os pesos à desmama e pós-desmama de bovinos da raça Tabapuã no Brasil. Revista Brasileira de Zootecnia, v.31, n.2, p.635-640, 2002.

GELMAN, A.; CARLIN, J.B.; STERN, H.S.; RUBIN, D.B. Bayesian data analysis. $2^{\text {nd }}$ ed. Boca Raton: Chapman \& Hall, 2004. 668p.

GIANNOTTI, J. Di G.; PACKER, I.U.; MERCADANTE, M.E.Z. Meta-análise para estimativas de correlação genética entre pesos ao nascer e desmama de bovinos. Scientia Agricola, v.59, p.435-440, 2002.

GILKS, W.R.; THOMAS, A.; SPIEGELHALTER, D.J. A language and program for complex Bayesian modelling. The Statistician, v.43, p.169-177, 1994.

KOOTS, K.R.; GIBSON, J.P.; SMITH, C.; WILTON, J.W. Analyses of published genetic parameter estimates for beef production traits. I. Heritability. Animal Breeding Abstracts, v.62, p.309-338, 1994a. 
KOOTS, K.R.; GIBSON, J.P.; SMITH, C.; WILTON, J.W. Analyses of published genetic parameter estimates for beef production traits. II. Phenotypic and genetic correlations. Animal Breeding Abstracts, v.62, p.826-853, 1994b.

LAROSE, D.T.; DEY, D.K. Grouped random effects models for bayesian meta-analysis. Statistics in Medicine, v.16, p.1817-1829, 1997.

LÔBO, R.N.B.; MADALENA, F.E.; VIEIRA, A.R. Average estimates of genetic parameters for beef and dairy cattle in tropical regions. Animal Breeding Abstracts, v.68, p.433-462, 2000.

MERCADANTE, M.E.Z.; LÔBO, R.B.; REYES, A. Parámetros genéticos para características de crecimiento en cebuínos de carne: una revisión. Archivo Latinoamericano de Producción Animal, v.3, p.45-89, 1995.

MEYER, K. Estimates of genetic parameters for weaning weight of beef cattle accounting for direct-maternal environmental covariances. Livestock Production Science, v.52, p.187-199, 1997.

MOHIUDDIN, G. Estimates of genetic and phenotypic parameters of some traits in beef cattle. Animal Breeding Abstracts, v.61, p.495-522, 1993.

NORMAND, S.T. Tutorial in biostatistics meta-analysis: formulating, evaluating, combining, and reporting. Statistics in Medicine, v.18, p.321-359, 1999.

PLASSE, D.; VERDE, O.S.; ARANGO, J.; CAMARIPANO, L.; FOSSI, H.; ROMERO, R.; RODRIGUEZ, M.C.; RUMBOS, J.L. (Co)variance components, genetic parameters and annual trends for calf weights in a Brahman herd kept on floodable savanna. Genetics and Molecular Research, v.1, p.282-297, 2002.
R DEVELOPMENT CORE TEAM. R: a language and environment for statistical computing. Disponível em: <http://www.rproject.org>. Acesso em: 2 jun. 2003.

RIBEIRO, M.N.; PIMENTA FILHO, E.C.; MARTINS, G.A.; SARMENTO, J.L.R.; MARTINS FILHO, R. Herdabilidade para efeitos direto e materno de características de crescimento de bovinos nelore no Estado da Paraíba. Revista Brasileira de Zootecnia, v.30, p.1224-1227, 2001.

SAKAGUTI, E.S. Funções de covariâncias e modelos de regressão aleatória na avaliação genética do crescimento de bovinos jovens da raça Tabapuã. 2000. 86p. Tese (Doutorado) Universidade Federal de Viçosa, Viçosa.

SCHAEFFER, L.R. Animal models-10-637-winter 99. Guelph: University of Guelph, 1999.

SMITH, T.C.; SPIEGELHALTER, D.J. Bayesian meta-analysis of randomized trials using graphical models and BUGS. In: BERRY, D.A.; STANGL, D.K. Bayesian biostatistics. New York: M. Dekker, 1996. p.411-427.

SPIEGELHALTER, D.J.; THOMAS, A.; BEST, N.G.; GILKS, W.R. BUGS: Bayesian inference using Gibbs sampling, version 0.50 . Disponível em: <http://www.mrc-bsu.cam.ac.uk/bugs/> . Acesso em: 2 jun. 2004.

TAWAH, C.L.; MBAH, D.A.; REGE, J.E.O.; OUMATE, H. Genetic evaluation of birth and weaning weight of Gudali and two-breed synthetic Wakwa beef cattle populations under selection in Cameroon: genetic and phenotypic parameters. Animal Production, v.57, p.73, 1993.

$\overline{\text { Recebido em } 29 \text { de setembro de } 2004 \text { e aprovado em } 4 \text { de maio de } 2005}$ 